\title{
Implementation of Empagliflozin in Patients with Diabetes Mellitus Type 2 and Established Cardiovascular Disease: Estimation of 5-Year Survival and Costs in Sweden
}

\author{
Lars Bernfort (1) - Magnus Husberg · Ann-Britt Wiréhn • \\ Ulf Rosenqvist · Staffan Gustavsson • Kristina Karlsdotter • \\ Lars-Åke Levin
}

Received: August 26, 2020 / Accepted: September 28, 2020 / Published online: October 6, 2020

(C) The Author(s) 2020

\begin{abstract}
Introduction: Cardiovascular disease (CVD) affects approximately $30 \%$ of patients with diabetes mellitus type 2 (T2D) and leads to increased morbidity, decreased survival and increased healthcare utilization. The aim of this study was to estimate the impact of treating these patients with the sodium-glucose cotransporter 2 (SGLT2) inhibitor empagliflozin on survival and healthcare utilization.
\end{abstract}

Methods: Actual survival and healthcare utilization data from a 5-year retrospective cohort

L. Bernfort $(\bowtie) \cdot$ M. Husberg · Lars-ÅkeLevin Department of Health, Medicine and Caring Sciences, Linköping University, Linköping, Sweden e-mail: lars.bernfort@liu.se

\section{A.-B. Wiréhn}

Research and Development Unit in Region

Östergötland, and Department of Health, Medicine and Caring Sciences, Linköping University,

Linköping, Sweden

U. Rosenqvist

Department of Internal Medicine, Motala Hospital,

Motala, Sweden

S. Gustavsson · K. Karlsdotter

Boehringer Ingelheim AB, Stockholm, Sweden study on patients with T2D and CVD in the Region of Östergötland, Sweden were used as a starting point. Actual data were adjusted in accordance with risk reductions for mortality and CV events related to empagliflozin treatment as reported in the EMPA-REG OUTCOME study.

Results: Applying the risk reductions related to empagliflozin treatment on the cohort of patients with T2D and CVD in Östergötland resulted in an increase in 5-year survival of 96 days per patient and reduced costs for healthcare and drugs other than empagliflozin. Including the cost of empagliflozin, treatment led to an increased net cost per patient of approximately SEK 18,000 over 5 years.

Conclusion: Empagliflozin treatment would reduce mortality and healthcare utilization in the patient group. The treatment strategy should be considered cost-effective, supporting a broad implementation of empagliflozin for patients with T2D and established CVD, in line with current national and international guidelines.

Keywords: Cardiovascular disease; Disease burden; Healthcare costs; Mortality; Pharmaceutical treatment; Register study; SGLT2 inhibitors; Type 2 diabetes 


\section{Key Summary Points}

Cardiovascular disease is common and constitutes a substantial disease burden in patients with type 2 diabetes, in terms of both mortality and healthcare-demanding events.

Modern glucose-lowering treatments, such as SGLT2 inhibitors, have a protecting effect for patients with type 2 diabetes and concomitant cardiovascular disease.

Applying results from the EMPA-REG OUTCOME study on a register-based cohort of patients, this study aimed to estimate the impact on survival and healthcare consumption of treating patients with type 2 diabetes and concomitant cardiovascular disease with the SGLT2 inhibitor empagliflozin.

The results of this study indicate that treatment with empagliflozin of patients with type 2 diabetes and cardiovascular disease is associated with both survival gains and lower healthcare costs.

Patients with type 2 diabetes and established cardiovascular disease would gain substantially from treatment with empagliflozin, and therefore more patients should receive this treatment.

\section{DIGITAL FEATURES}

This article is published with digital features, including a summary slide, to facilitate understanding of the article. To view digital features for this article go to https://doi.org/10.6084/ m9.figshare.13013333.

\section{INTRODUCTION}

Type 2 diabetes mellitus (T2D) is a major global public health problem with a prevalence of
$4-5 \%[1,2]$. T2D is associated with impaired quality of life and a substantial economic burden due to high healthcare costs and utilization. A common comorbidity in patients with T2D is cardiovascular disease (CVD). The reported prevalence of CVD in patients with T2D in Sweden is 28.3\% [3]; it is slightly higher in the region of Östergötland, at 30\% [4]. Östergötland is a public healthcare region in the southeast of Sweden with approximately 460,000 residents, i.e. $4-5 \%$ of the Swedish population. Östergötland demographics (e.g. age, gender, etc.) are representative of Sweden as a whole. The Swedish healthcare system is primarily publicly financed, and healthcare services are available to all residents.

T2D and associated comorbidities, including CVD, lead to increased risk of complications, which in turn lead to increased burden of disease and healthcare utilization and costs. Patients with T2D and established CVD are more likely to have negative healthcare consequences and higher healthcare costs compared to patients with T2D without established CVD [4]. This CVD exposure combined with the high prevalence of T2D makes it clear how important prioritizing well-informed healthcare-related decisions is in this patient group.

On the basis of growing evidence, treatment of patients with T2D and established CVD with sodium-glucose cotransporter 2 (SGLT2) inhibitors or glucagon-like peptide 1 (GLP-1) receptor agonists is recommended by the Swedish National Board of Health and Welfare [2], the European Society of Cardiology (ESC) [5], as well as by the joint statement from the American Diabetes Association (ADA) and the European Association for the Study of Diabetes (EASD) [6]. In addition to their glucose-lowering effect, these drugs also lower the risk for CVD $[7,8]$, and slow the progression of nephropathy [9].

The SGLT2 inhibitor empagliflozin, in addition to being part of the standard of care for patients with T2D and established CVD, was studied with respect to its effect on mortality and selected cardiovascular outcomes in the EMPA-REG OUTCOME study [10]. It was found that empagliflozin reduced death from any cause during the study period $(5.7 \%$ of patients 
taking empagliflozin died of any cause vs $8.3 \%$ of patients on placebo; $32 \%$ relative risk reduction), death from CV causes $(3.7 \%$ and $5.9 \%$, respectively; $38 \%$ relative risk reduction) and the proportion of patients hospitalised for heart failure $(2.7 \%$ and $4.1 \%$, respectively; $35 \%$ relative risk reduction).

In a previous study conducted using a dataset from before the more widespread use of SGLT2 inhibitors in routine care, we found that established CVD was associated with higher morbidity, mortality and healthcare costs in patients with T2D [4]. There is a growing body of real-world evidence demonstrating the beneficial effects of prescribing empagliflozin to patients with T2D and established CVD in terms of its impact on healthcare utilization, costs and survival $[11,12]$.

The aim of this study was to estimate effects on survival, healthcare utilization and cost of 5 years of treatment with empagliflozin, in addition to standard of care, in patients with T2D and established CVD, in a Swedish realworld setting.

\section{METHODS}

To estimate the effect on health outcomes related to the implementation of empagliflozin in accordance with published national guidelines from the Swedish National Board of Health and Welfare, the risk reductions as identified in the EMPA-REG OUTCOME study were applied to the T2D CVD cohort in the region of Östergötland, as described below.

The study was approved by the Regional Ethics Research Committee in Östergötland, Sweden (Dnr: 2017/535-31). The study was performed in accordance with the Helsinki Declaration of 1964 and its later amendments. Informed consent was not required because of the retrospective nature of the study.

\section{T2D CVD Cohort}

The T2D CVD cohort consists of all patients with T2D and established CVD in the healthcare region of Östergötland. With few exceptions, patients with T2D in Sweden are treated within the primary care setting. Only patients with acute diabetes or undergoing a complex endocrine investigation might be hospitalised. Healthcare utilization for the Östergötland cohort was retrieved from the administrative database of Region Östergötland (Healthcare Data Warehouse, VDL). The Östergötland VDL has almost $100 \%$ coverage and contains information on all healthcare utilization (primary care visits, outpatient visits and inpatient hospital stays) for all residents in the region. Drug utilization was retrieved from the Swedish Prescribed Drug Register.

The T2D CVD cohort in Östergötland and the data sources used were described in detail elsewhere [4]. This was a population-based retrospective observational study which analysed historical data on healthcare utilization, costs and mortality among patients with T2D with and without established CVD for the period between 2012 and 2016. In this previous study, briefly, we found that in the region of Östergötland there were 19,731 patients with T2D (4.5\% prevalence). The cohort identified for this analysis consisted of all patients with T2D and established CVD by 1 January 2012 which was 5490 patients. Established CVD was defined according to inclusion and exclusion criteria in the EMPA-REG OUTCOME study [10], which included any of the following diagnoses any time during the period 2002-2011: coronary artery disease (CAD), stroke, amputation, percutaneous coronary intervention (PCI), coronary artery bypass grafting (CABG), peripheral artery disease (PAD), cardiac arrest and angioplasty (carotid or femoral). Exclusion criteria from the EMPA-REG OUTCOME study [10] were also applied: < 18 years, body mass index $>45 \mathrm{~kg} / \mathrm{m}^{2}$, severely impaired renal function (GFR $<30 \mathrm{~mL} / \mathrm{min})$ and recent (within 2 months prior to study baseline) stroke, transient ischemic attack (TIA) or acute coronary syndrome (ACS).

The study cohort was followed retrospectively for 5 years (2012-2016). Data on actual outcomes in terms of mortality, events requiring healthcare, healthcare utilization and costs were retrieved from registers including the Swedish Cause of Death Register, the Swedish Prescribed Drug Register, the National Diabetes 
Register and the administrative database of Region Östergötland (VDL). Detailed information on data sources used and healthcare costs associated with various outcomes during the 5 years of follow-up in the Östergötland cohort was provided in a previous publication [4].

Costs in this manuscript are expressed in Swedish krona (SEK). Exchange rates according to the Swedish National Bank as of August 2020 were 1 euro $=10.31$ SEK; 1 United States dollar $(\mathrm{USD})=8.71 \mathrm{SEK}$.

\section{Application of Relative Risks from EMPA- REG OUTCOME Study}

To estimate the effects on costs and survival of 5 years of treatment with empagliflozin in the relevant patient group in Östergötland, the relative risks observed in the EMPA-REG OUTCOME study were applied to the T2D CVD cohort.

\section{Statistical Analysis}

The effects of treating the T2D CVD cohort with empagliflozin were estimated using the assumption that the effects in a real-world setting are the same as those found in the EMPAREG OUTCOME study. These effects were expressed as relative risk ratios (RR) and hazard ratios (HR). For costs and healthcare utilization, the RRs were used for the whole study period, and survival changes were estimated by applying the HR from the EMPA-REG OUTCOME study to the life tables of the patients in the T2D CVD cohort (Table 1 ).

\section{RESULTS}

\section{Baseline Characteristics}

Some differences were found in the baseline characteristics of the EMPA-REG OUTCOME study population and the T2D CVD cohort respectively.

As shown in Table 2, the T2D CVD cohort was older (73 vs 63 years), contained more women ( $42 \%$ and $28 \%$ respectively), had fewer patients with long T2D duration ( $>10$ years; $51 \%$ and $57 \%$ respectively), had lower HbA1c values (54 vs 64) and had on average worse kidney function than the patients in the EMPAREG OUTCOME study. Furthermore, a larger proportion of the EMPA-REG OUTCOME study population had previously experienced MI or PAD, and fewer patients in the Östergötland cohort were treated with insulin. Proportion of patients being treated for CVD risk factors (blood pressure, lipids, etc.) was as expected. In addition to the baseline differences between the cohorts, there was also a difference in the length of follow-up: 5 years for the T2D CVD cohort,

Table 1 Relative risks for events and hazard ratio for death as observed in the EMPA-REG OUTCOME study [10]

\begin{tabular}{llllr}
\hline & Placebo (\%) & Empagliflozin (\%) & RR & $\boldsymbol{p}$ value \\
\hline Myocardial infarction (MI) & 5.4 & 4.8 & 0.88 & 0.243 \\
Unstable angina & 2.8 & 2.8 & 1.00 & 0.984 \\
Coronary revascularisation & 8 & 7 & 0.88 & 0.149 \\
Stroke & 3 & 3.5 & 1.18 & 0.233 \\
Transient ischaemic attack (TIA) & 1 & 0.8 & 0.84 & 0.517 \\
Heart failure & 4.1 & 2.7 & 0.66 & 0.002 \\
Dialysis [21] & 0.6 & 0.3 & 0.46 & 0.040 \\
Death $^{\text {a }}$ & & & $0.68^{\text {a }}$ & $<0.001$ \\
\hline
\end{tabular}

a The comparison concerning death is expressed as hazard ratio (HR) 
Table 2 Baseline characteristics in the Östergötland T2D CVD cohort and in the EMPA-REG study population (mean, $\%)$

\begin{tabular}{|c|c|c|c|}
\hline & T2D CVD & EMPA-REG & $p$ value \\
\hline Age & 73.5 & 63.1 & 0.000 \\
\hline Sex (\% men) & $58.10 \%$ & $71.20 \%$ & 0.000 \\
\hline BMI & 29.8 & 30.6 & 0.000 \\
\hline Weight & 85.1 & 86.2 & 0.001 \\
\hline \multicolumn{4}{|l|}{$\mathrm{CV}$ risk factors (history of) } \\
\hline CAD & $77.40 \%$ & $75.60 \%$ & 0.028 \\
\hline MI & $26.10 \%$ & $46.70 \%$ & 0.000 \\
\hline Revascularisation & $27.00 \%$ & $25.10 \%$ & 0.034 \\
\hline Stroke & $24.80 \%$ & $23.10 \%$ & 0.049 \\
\hline PAD & $14.50 \%$ & $21.00 \%$ & 0.000 \\
\hline Diabetes duration & & & 0.000 \\
\hline$\leq 5$ years & $23.90 \%$ & $17.90 \%$ & \\
\hline $5-10$ years & $24.90 \%$ & $25.10 \%$ & \\
\hline$>10$ years & $51.20 \%$ & $57.00 \%$ & \\
\hline HbAlc & 54 & 64.7 & 0.000 \\
\hline LDL & 2.3 & 2.2 & 0.000 \\
\hline Systolic blood pressure & 133 & 135.3 & 0.000 \\
\hline Diastolic blood pressure & 72.2 & 76.6 & 0.000 \\
\hline GFR & & & 0.000 \\
\hline$\geq 90$ & $16.00 \%$ & $22.40 \%$ & \\
\hline 60 to $<90$ & $47.30 \%$ & $51.70 \%$ & \\
\hline$<60$ & $36.70 \%$ & $25.90 \%$ & \\
\hline Albuminuria & & & 0.000 \\
\hline Normal value & $68.60 \%$ & $60.20 \%$ & \\
\hline Micro albuminuria & $20.20 \%$ & $28.90 \%$ & \\
\hline Macro albuminuria & $11.20 \%$ & $11.00 \%$ & \\
\hline \multicolumn{4}{|c|}{ Medication within 3 months after 1 January 2012} \\
\hline Insulin (A10A) & $34.70 \%$ & $48.00 \%$ & 0.000 \\
\hline A10BA Biguanide derivates (metformin) & $31.20 \%$ & $73.80 \%$ & 0.000 \\
\hline A10BB Sulfonylurea compounds & $10.10 \%$ & $43.00 \%$ & 0.000 \\
\hline A10BG Thiazolidinediones & $0.20 \%$ & $4.20 \%$ & 0.000 \\
\hline A10BH Dipeptidyl peptidase 4 inhibitors & $1.20 \%$ & $11.30 \%$ & 0.000 \\
\hline
\end{tabular}


Table 2 continued

\begin{tabular}{llcc}
\hline & T2D CVD & EMPA-REG & $\boldsymbol{p}$ value \\
\hline A10BJ Glucagon-like peptide 1 receptor (GLP-1) analogues & $0.60 \%$ & $2.70 \%$ & 0.000 \\
\hline
\end{tabular}

Table 3 Mean healthcare costs (SEK) and cost changes during 5 years with and without the addition of empagliflozin to patients with T2D and established CVD

\begin{tabular}{lrlrr}
\hline & $\begin{array}{l}\text { Östergötland cohort } \\
\text { costs }\end{array}$ & $\begin{array}{l}\text { RR according to EMPA- } \\
\text { REG }\end{array}$ & $\begin{array}{l}\text { Costs adjusted with } \\
\text { RR }\end{array}$ & Difference \\
\hline CV care & & & 863 & -108 \\
Myocardial infarction & 971 & 0.89 & 459 & 0 \\
Unstable angina & 459 & 1.00 & 5318 & -725 \\
Coronary & 6043 & 0.88 & & 409 \\
revascularisation & & & 2866 & -21 \\
Stroke & 2457 & 1.17 & 103 & -2342 \\
TIA & 125 & 0.83 & 4517 & -1355 \\
Heart failure & 6859 & 0.66 & 1164 & 0 \\
Dialysis & 2519 & 0.46 & 156,443 & -4142 \\
Other healthcare & 156,443 & 1 & 171,733 & 23,910 \\
Healthcare total & 175,875 & & 23,910 & -1791 \\
Empagliflozin & 0 & - & 44,750 & 22,119 \\
Other drugs & 46,541 & - & 68,660 & 17,977 \\
Drugs total & 46,541 & - & 240,393 & \\
Total cost & 222,416 & &
\end{tabular}

compared to the 3.1 years median follow-up in the EMPA-REG OUTCOME study.

The effect of widespread utilization of empagliflozin in patients with T2D and established CVD (if all patients in this cohort were treated with empagliflozin) is described in Table 3.

The most substantial health effects associated with empagliflozin treatment observed in the EMPA-REG OUTCOME study were reduced mortality (HR 0.68), fewer hospitalisations due to heart failure (RR 0.66), fewer revascularisations (RR 0.88) and a reduction in dialysis (RR 0.46). Owing to the beneficial health effects related to empagliflozin use, the estimation of the effect of adding empagliflozin to standard treatment for patients with T2D and established CVD resulted in reduced average costs for healthcare (- SEK 4142 of which SEK 2342 was related to heart failure) and other drugs (- SEK 1791). The cost of empagliflozin treatment was estimated to be SEK 23,910 per patient, which amounted to net increased costs of SEK 17,977 per patient. 
The positive treatment effects of empagliflozin, e.g. the reduced risk of CV events, result in decreased mortality in the patient group. During the 5-year treatment period, the estimated mean survival increased from 1498 days to 1594 days, a survival gain of 96 days per treated patient. Estimated survival gains with empagliflozin are described in Fig. 1 and Table 4.

\section{DISCUSSION}

This register-based retrospective cohort study aimed to estimate the consequences of a widereaching implementation of the SGLT2 inhibitor empagliflozin for patients with T2D and established CVD. Though there are other novel glucose-lowering medications, this study was limited to empagliflozin, as it is the recommended treatment according to regional (Östergötland) and national (Sweden) guidelines. Risk reductions regarding cardiovascular events and mortality as identified in the EMPAREG OUTCOME study were applied on a geographically defined cohort of patients with T2D and established CVD, in the region of Östergötland, Sweden (the T2D CVD cohort). At baseline, i.e. during the first 3 months of 2012, $85 \%$ of the patients in the T2D CVD cohort received antihypertensive treatment and 58\%

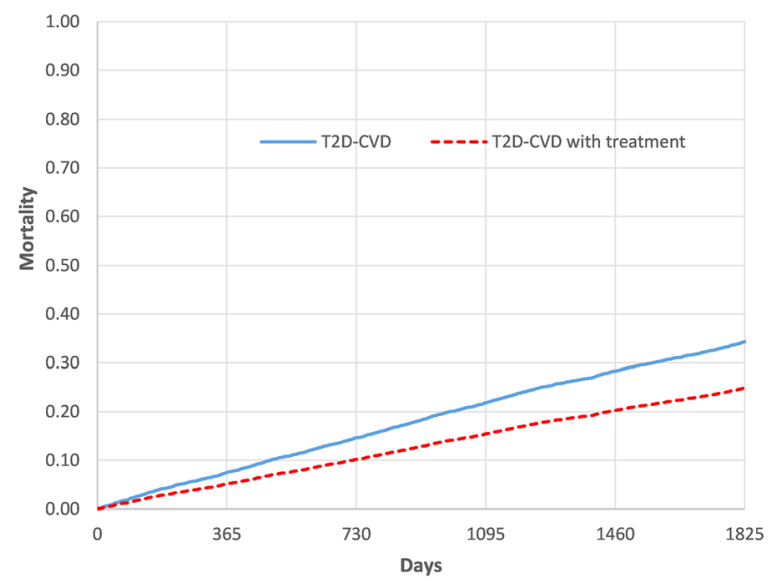

Fig. 1 Five-year cumulative mortality in the T2D CVD cohort, actual outcome and adjusted according to the results in the EMPA-REG OUTCOME study $(\mathrm{HR}=0.68)$ received lipid-lowering treatment, indicating that there was room for improvement according to the national guidelines. The effects of applying the EMPA-REG OUTCOME study risk reductions to all patients with T2D and established CVD during a 5-year period included reduced healthcare utilization and improved survival, at a low cost. The results suggest that utilization of empagliflozin for patients with T2D and established CVD would be a good use of healthcare resources. The estimated decrease in the costs of healthcare visits was approximately SEK 4100; increasing drug costs (for empagliflozin) resulted in a net cost increase of approximately SEK 18,000. This increase should be evaluated in the context of the survival gain of 96 days per treated patient, over a period of 5 years.

The results from this study indicate that the increased empagliflozin utilization would cost approximately SEK 69,000 per life-year gained. Applying a quality of life decrement of 0.19 (on a scale 0-1) for patients with T2D and established CVD [13] the result would translate to a cost per quality-adjusted life-year (QALY) of just over SEK 85,000. These preliminary results should be interpreted with caution as we have not applied a lifetime perspective on survival and costs. Our findings, however, indicate that empagliflozin is cost-effective, as the estimated cost per QALY is well below both commonly applied threshold values [14] and the marginal productivity of the healthcare sector [15].

For the public healthcare region of Östergötland, 5 years of treatment with empagliflozin for the 5490 patients with T2D and established CVD identified in this study would result in increased costs of approximately SEK 100 million (SEK 20 million per year), and 1455 life-years gained (approximately 290 lifeyears per year of treatment). Extrapolated to the country level, each year of treatment with empagliflozin would result in approximately 5800 life-years gained at a cost of approximately SEK 440 million.

Our results indicate that treating this vulnerable patient population with empagliflozin has positive effects on health and is also costeffective. Treatment of this patient group should follow international and national 
Table 4 Estimated number of lived and lost days with and without 5 years empagliflozin treatment

\begin{tabular}{llcc}
\hline & $\begin{array}{l}\text { Maximum number of days during the } \\
\text { period }\end{array}$ & $\begin{array}{l}\text { Living } \\
\text { days }\end{array}$ & $\begin{array}{c}\text { Lost } \\
\text { days }\end{array}$ \\
\hline T2D CVD cohort & 1826 & 1498 & 328 \\
T2D CVD cohort adjusted according to EMPA- & 1826 & 1594 & 232 \\
REG & & & -96 \\
Benefit with empagliflozin treatment & & 96 & -96 \\
\hline
\end{tabular}

guidelines [2, 5]. Previous research shows that the implementation and uptake of new diabetes medications in Sweden is generally slow, placing it in this respect far behind its neighbouring countries [16].

The main strength of the study is that it is based on patient data from high-quality registers, which means that the mapping of the cohort from Östergötland is very reliable. Information regarding healthcare utilization, drug consumption, costs and mortality of this patient cohort from 2012 to 2016 are well recorded in these registers.

Previous research has shown that glucoselowering drugs for patients with T2D and established CVD have a beneficial effect with a reduced risk of cardiovascular events [7, 8, 17]. The beneficial effects are particularly noticeable for SGLT2 inhibitors [8]. Costs associated with the treatment of patients with T2D and established CVD have been studied to a lesser extent. A study conducted in the USA [18] compared two different glucose-lowering drugs, one SGLT2 inhibitor (dapagliflozin) and the other a dipeptidyl peptidase 4 inhibitor (sitagliptin). It was concluded that dapagliflozin led to higher drug costs but that this was offset by lower healthcare costs. In a Swedish registry-based study [19], dapagliflozin was compared with "other glucose-lowering drugs" (other SGLT2 inhibitors excluded). This study also concluded that dapagliflozin reduced hospital care costs related to CVD, while also leading to higher drug costs for patients with T2D and established CVD or multiple risk factors for CVD. Comparing different SGLT2 inhibitors, it has been suggested that empagliflozin prescribed for preventing $\mathrm{CV}$ death and hospitalisation for heart failure in patients with T2D is cost-effective compared to treatment with canagliflozin or dapagliflozin [20].

\section{Limitations}

Our results are associated with some uncertainty. Even though the inclusion criteria of the EMPA-REG OUTCOME study were applied when including patients in the T2D CVD cohort in Östergötland, some differences between the populations were apparent. For example, the Östergötland T2D CVD cohort was older than the patients in the EMPA-REG OUTCOME study and contained a greater proportion of women. Although the CVD cohort in Östergötland was older, it included a lower proportion of patients with long ( $>10$ years) diabetes duration and, on average, patients had lower HbA1c values compared to the EMPA-REG OUTCOME study population. However, the Östergötland patients had poorer kidney function, which is reasonable given the age difference. As a result of these differences the study should be regarded as exploratory only.

Caution should be exercised when it comes to the generalizability of results reported in this study. Results are specific to patients with T2D and established CVD and should not be extrapolated to patients with T2D without established CVD (primary prevention). As the study was conducted in Sweden, there may be differences in this patient population that would make generalizing the results to other countries problematic, such as differences in patient population characteristics, national treatment guidelines and therapeutic treatment in $\mathrm{T} 2 \mathrm{D}$. 


\section{CONCLUSION}

Results from this study, based on all patients in Östergötland with T2D and established CVD in 2012, and results from the EMPA-REG OUTCOME study, show that empagliflozin treatment would reduce mortality and the need for healthcare in the patient group. Adding the cost for empagliflozin, the treatment strategy should still be considered cost-effective, supporting a broad implementation of empagliflozin (in addition to standard treatment) to patients with T2D and established CVD. Our results emphasise the importance of following current national and international guidelines.

\section{ACKNOWLEDGEMENTS}

Funding. This study was sponsored by a grant from Boehringer Ingelheim AB, Sweden. The journal's Rapid Service Fee was funded by the Linköping University Library.

Authorship. All named authors meet the International Committee of Medical Journal Editors (ICMJE) criteria for authorship for this article, take responsibility for the integrity of the work as a whole, and have given their approval for this version to be published.

Disclosures. Lars Bernfort, Magnus Husberg, Ann-Britt Wiréhn, Ulf Rosenqvist and Lars-Åke Levin have nothing to disclose. Staffan Gustavsson and Kristina Karlsdotter are employed by Boehringer Ingelheim AB.

Compliance with Ethics Guidelines. The study was approved by the Regional Ethics Research Committee in Östergötland, Sweden (Dnr: 2017/535-31). The study was performed in accordance with the Helsinki Declaration of 1964 and its later amendments. Informed consent was not required because of the retrospective nature of the study.

Data Availability. The datasets generated during and/or analyzed during the current study are available from the corresponding author on reasonable request.

Open Access. This article is licensed under a Creative Commons Attribution-NonCommercial 4.0 International License, which permits any non-commercial use, sharing, adaptation, distribution and reproduction in any medium or format, as long as you give appropriate credit to the original author(s) and the source, provide a link to the Creative Commons licence, and indicate if changes were made. The images or other third party material in this article are included in the article's Creative Commons licence, unless indicated otherwise in a credit line to the material. If material is not included in the article's Creative Commons licence and your intended use is not permitted by statutory regulation or exceeds the permitted use, you will need to obtain permission directly from the copyright holder. To view a copy of this licence, visit http:// creativecommons.org/licenses/by-nc/4.0/.

\section{REFERENCES}

1. Huebschmann AG, Huxley RR, Kohrt WM, Zeitler P, Regensteiner JG, Reusch JEB. Sex differences in the burden of type 2 diabetes and cardiovascular risk across the life course. Diabetologia. 2019;62(10): 1761-72.

2. Swedish National Board of Health and Welfare, National Guidelines for Diabetes Care-Support for governance and management. Stockholm; 2018

3. Eliasson B, Ekelund J, Amberntsson R, Miftaraj M, Svensson AM. Cardiovascular disease in patients with type 2 diabetes and in patients starting empagliflozin treatment: nationwide survey. Diabetes Ther. 2019;10(4):1523-30.

4. Bernfort L, Husberg M, Wirehn AB, et al. Disease burden and healthcare costs for T2D patients with and without established cardiovascular disease in Sweden: a retrospective cohort study. Diabetes Ther. 2020;11:1537-49.

5. Cosentino F, Grant PJ, Aboyans V, et al. 2019 ESC Guidelines on diabetes, pre-diabetes, and cardiovascular diseases developed in collaboration with the EASD. Eur Heart J. 2020;41(2):255-32323. 
6. Buse JB, Wexler DJ, Tsapas A, et al. 2019 Update to: Management of Hyperglycemia in Type 2 Diabetes, 2018. A Consensus Report by the American Diabetes Association (ADA) and the European Association for the Study of Diabetes (EASD). Diabetes Care. 2020;43(2):487-93.

7. Kristensen SL, Rorth R, Jhund PS, et al. Cardiovascular, mortality, and kidney outcomes with GLP-1 receptor agonists in patients with type 2 diabetes: a systematic review and meta-analysis of cardiovascular outcome trials. Lancet Diabetes Endocrinol. 2019;7(10):776-85.

8. Zelniker TA, Wiviott SD, Raz I, et al. SGLT2 inhibitors for primary and secondary prevention of cardiovascular and renal outcomes in type 2 diabetes: a systematic review and meta-analysis of cardiovascular outcome trials. Lancet. 2019;393(10166):31-9.

9. Perkovic V, Jardine MJ, Neal B, et al. Canagliflozin and renal outcomes in type 2 diabetes and nephropathy. N Engl J Med. 2019;380(24): 2295-306.

10. Zinman B, Wanner C, Lachin JM, et al. Empagliflozin, cardiovascular outcomes, and mortality in type 2 diabetes. N Engl J Med. 2015;373(22): 2117-288.

11. Patorno E, Pawar A, Franklin JM, et al. Cardiovascular effectiveness of empagliflozin compared to DPP4 inhibitors and to GLP1 receptor agonists: interim analysis from the EMPagliflozin compaRative effectIveness and SafEty (EMPRISE) study. Circulation. 2019;140(Suppl 1):Abstract 11928.

12. Najafzadeh M, Pawar A, Schneeweiss S, et al. Healthcare resource utilization among empagliflozin initiators with and without cardiovascular disease versus DPP4 $i$ in a commercially-insured routine care population: an analysis from the EMPagliflozin compaRative effectIveness and SafEty (EMPRISE) study. Circulation. 2019;140(Suppl 1): Abstract 13655.

13. Levin LA, Wallentin L, Bernfort L, et al. Health-related quality of life of ticagrelor versus clopidogrel in patients with acute coronary syndromes-results from the PLATO trial. Value Health. 2013;16(4): 574-80.

14. Swedish National Board of Health and Welfare, National Guidelines for the Diabetes Care 2010Support for governance and management, Appendix 4, Method. Stockholm; 2010.

15. Siverskog J, Henriksson M. Estimating the marginal cost of a life year in Sweden's public healthcare sector. Eur J Health Econ. 2019;20(5):751-62.

16. Persson F, Bodegard J, Lahtela JT, et al. Different patterns of second-line treatment in type 2 diabetes after metformin monotherapy in Denmark, Finland, Norway and Sweden (D360 Nordic): a multinational observational study. Endocrinol Diabetes Metab. 2018;1(4):e00036.

17. Santamarina M, Carlson CJ. Review of the cardiovascular safety of dipeptidyl peptidase-4 inhibitors and the clinical relevance of the CAROLINA trial. BMC Cardiovasc Disord. 2019;19(1):60. https://doi. org/10.1186/s12872-019-1036-0.

18. Parker ED, Wittbrodt ET, McPheeters JT, Frias JP. Comparison of healthcare resource utilization and costs in patients with type 2 diabetes initiating dapagliflozin versus sitagliptin. Diabetes Obes Metab. 2019;21(2):227-33.

19. Norhammar A, Bodegard J, Nystrom T, et al. Dapagliflozin vs non-SGLT-2i treatment is associated with lower healthcare costs in type 2 diabetes patients similar to participants in the DECLARETIMI 58 trial: a nationwide observational study. Diabetes Obes Metab. 2019. https://www.ncbi.nlm. nih.gov/pubmed/31379124. Accessed 5 Aug.

20. Arbel R, Aboalhasan E, Hammerman A, Azuri J. Sodium-glucose cotransporter 2 inhibitors for prevention of heart failure events in patients with type 2 diabetes mellitus: a cost per outcome analysis. Clin Drug Investig. 2020;40(7):665-9.

21. Wanner C, Inzucchi SE, Lachin JM, et al. Empagliflozin and progression of kidney disease in type 2 diabetes. N Engl J Med. 2016;375(4):323-34. 